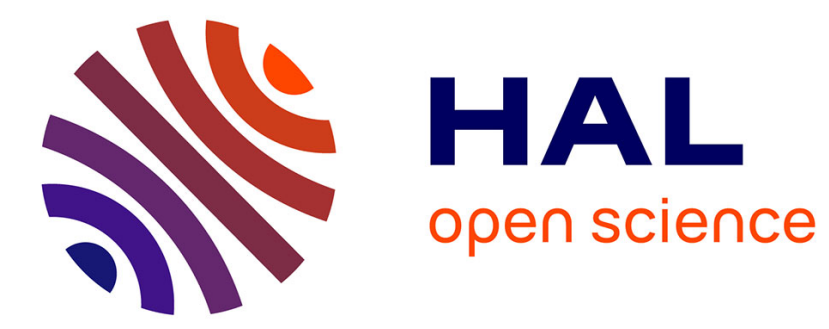

\title{
Closures on partial partitions from closures on sets
}

Christian Ronse

\section{To cite this version:}

Christian Ronse. Closures on partial partitions from closures on sets. Mathematica Slovaca, 2013, 63 (5), 10.2478/s12175-013-0147-9 . hal-02882871

\section{HAL Id: hal-02882871 https://hal.science/hal-02882871}

Submitted on 17 Jul 2020

HAL is a multi-disciplinary open access archive for the deposit and dissemination of scientific research documents, whether they are published or not. The documents may come from teaching and research institutions in France or abroad, or from public or private research centers.
L'archive ouverte pluridisciplinaire HAL, est destinée au dépôt et à la diffusion de documents scientifiques de niveau recherche, publiés ou non, émanant des établissements d'enseignement et de recherche français ou étrangers, des laboratoires publics ou privés. 


\title{
CLOSURES ON PARTIAL PARTITIONS FROM CLOSURES ON SETS
}

\author{
CHRISTIAN RONSE
}

\begin{abstract}
Jordens and Sturm investigated the link between closure systems on sets and closure systems on partitions. We extend that study to the wider framework of partial partitions, and highlight better the relation between these two families of closure systems. Then we consider the construction of a closure operator on partial partitions by the iterated application a set operator to the blocks of a partial partition; the resulting closure system fits into our framework.
\end{abstract}

\section{IntRoduction}

A partial partition of a set $E$ is a partition of a subset of $E$, in other words a set of mutually disjoint non-void subsets of $E$ (called blocks). The refinement order on partitions [19] extends naturally to partial partitions, and the latter constitute then a complete lattice, which was briefly studied by Czekoslovak mathematicians 40 years ago [8, 9, 29].

Partitions or partial partitions are used in programming semantics [2, 18, 25], consensus theory (how to make a "median" classification from several empirically obtained classifications) [1, 13], and the management of spatial data. For example in clustering (unsupervised classification), several points in space are grouped on the basis of their mutual proximity relations or the shapes of various subgroups; the resulting classification is both a partition of the set of points and a partial partition of the whole space. The interest in the complete lattice of partitions was recently revived in the context of image analysis [24, 28], for the problem of image segmentation: to obtain from an image a partition of the underlying space, whose blocks represent the distinct homogeneous regions seen in the image; sometimes the regions do not cover the whole space (there is a

2010 Mathematics Subject Classification. Primary 03E02, 06A15, 06B99; Secondary $68 \mathrm{U} 10$.

Key words and phrases. partitions, partial partitions, complete lattice, closure operator, closure system.

This work received funding from the Agence Nationale de la Recherche, contract ANR2010-BLAN-0205-01. 
"background" or a set of separating borders between regions), and in this case we deal with the complete lattice of partial partitions [20, 22, 23].

An important topic in lattice theory is the study of maps with specific ordertheoretic properties, such as adjunctions $[5,7,12]$ or closure operators [10]. Besides their mathematical interest, such maps play a fundamental role in various applications of lattice theory to computer science, as they represent the processes operating on the data; we see this for example in programming semantics $[6,12]$, logic [4], formal concept analysis [11] and image analysis $[4,14,26]$.

Such specific operators are also important in the framework of the lattice of (partial) partitions. For instance in image segmentation, a partition is built either by splitting the space, or by successively clustering points; the two case correspond respectively to anti-extensive and extensive operators on partial partitions. The author recently investigated adjunctions [21], which are linked to hierarchies of segmentations, and idempotent anti-extensive operators that act by splitting each block [22, 23], the latter are used in several image segmentation approaches.

Jordens and Sturm $[16,17]$ studied closure operators on partitions in terms of the associated closure system (consisting of all invariants of the closure operator). For example given a closure system $\mathcal{S}$ on sets, the family of all partitions whose blocks belong to $\mathcal{S}$ will be a closure system on partitions; on the other hand, given a closure system $\Sigma$ on partitions, the set of all blocks of all members of $\Sigma$, with the empty set added, will be a closure system on sets. We deepen their investigation of this link between closure systems on sets and closure systems on partitions, and extend it to the case of partial partitions. Our approach relies on an adjunction between sets of parts of a space $E$ and sets of (partial) partitions of $E$.

If we apply a closure operator to the blocks of a partial partition and chain together the overlapping closed blocks, the resulting partial partition will generally have blocks that are not closed; then we can repeat the operation. Now a closure operator can be obtained as the limit of the iteration of an extensive and isotone operator satisfying some "continuity" condition [15]. We study thus the generation of a closure on partial partitions as the limit of the iteration of applying to the blocks a "continuous" extensive isotone operator $\psi$ on sets, and chaining the overlapping resulting blocks; the resulting closure system will consist of all partial partitions with blocks closed under the closure operator on sets that is the limit of the iteration of $\psi$.

In [21] it was shown that an adjunction on sets satisfying a "regularity" condition leads to an adjunction on partial partitions, by applying the operators to the blocks. We briefly investigate the relation between the two closures on sets and on partial partitions generated from these two adjunctions. 
The dual version of the topic of this paper has been investigated recently $[20,22,28]$ : for which type of family $\mathcal{S}$ of sets will the family of all (partial) partitions whose blocks belong to $\mathcal{S}$ be a dual closure system ? We recall the relevant results and discuss the combined problem of characterizing families $\mathcal{S}$ of sets for which the family of all (partial) partitions with blocks in $\mathcal{S}$ will be both a closure system and a dual closure system, i.e., a complete sublattice of the lattice of (partial) partitions.

A possible application of our results could be in clustering, if one requires the clusters to belong to a closure system, for example to be convex.

1.1. Paper organization. Subsection 1.2 gives our terminology and notation; it also recalls some essential facts about closure operators and systems, adjunctions, etc. Section 2 investigates the link between closure systems on sets and those on partitions or partial partitions, following the approach of Jordens and Sturm [16, 17]. Section 3 discusses the construction of closure operators on partial partitions from operators applying a given set operator to each block; we consider in particular iterations of extensive isotone operators and closures from adjunctions. Section 4 discusses our results and compares them with those of $[20,22,28]$ on dual closure systems of (partial) partitions; then it concludes.

1.2. Terminology and notation. Given two sets $A$ and $B$, we will write $(\alpha, \beta): A \rightleftharpoons B$, or say that $(\alpha, \beta)$ is $A \rightleftharpoons B$, if $\alpha$ is a map $A \rightarrow B$ and $\beta$ is $\operatorname{map} B \rightarrow A$.

We follow [14] for the notation concerning lattice operators. Let $L$ be a complete lattice with least element $\mathbf{0}$ and greatest element $\mathbf{1}$. We call an operator on $L$ any map $L \rightarrow L$. Operators will be designated by lower-case Greek letters $\alpha, \ldots, \omega$, except $\pi$, reserved for partial partitions; more specifically, we will use $\varphi$ for a closure, $\delta$ for a complete join-morphism, $\varepsilon$ for a complete meet-morphism, and $(\varepsilon, \delta)$ for an adjunction. We write $\psi(x)$ for the image of $x$ by $\psi$, and given two operators $\psi$ and $\xi$, the composition of $\psi$ followed by $\xi$, that is, the operator $x \mapsto \xi(\psi(x))$, will be written $\xi \psi$. For any integer $n \geq 1$, the power $\psi^{n}$ is the selfcomposition of $\psi n$ times; inductively, $\psi^{1}=\psi$ and $\psi^{n+1}=\psi^{n} \psi$. The invariance domain of an operator $\psi$ is the set $\operatorname{lnv}(\psi)=\{x \in L \mid \psi(x)=x\}$.

A closure operator on $L$ is an operator $\varphi$ that is isotone $(x \leq y \Rightarrow \varphi(x) \leq$ $\varphi(y))$, extensive $(x \leq \varphi(x))$ and idempotent $(\varphi(\varphi(x))=\varphi(x))$. A closure system on $L$ is a subset $S \subseteq L$ that is stable under the infimum operation (in particular for the empty infimum, $\mathbf{1} \in S)$. The map $\varphi \mapsto \operatorname{lnv}(\varphi)$ gives a dual isomorphism between the poset of closure operators and that of closure systems.

Clearly $\varphi(\mathbf{0})=\mathbf{0}$ iff $\mathbf{0} \in \operatorname{Inv}(\varphi)$. We call a 0 -closure system a closure system $S$ such that $\mathbf{0} \in S$, and a 0 -closure operator a closure operator $\varphi$ such that $\varphi(\mathbf{0})=\mathbf{0}$. Given a closure operator $\varphi$ and the corresponding closure system 
$S=\operatorname{lnv}(\varphi)$, then we obtain the 0-closure system $S_{0}=S \cup\{\mathbf{0}\}$, and $S_{0}=\operatorname{Inv}\left(\varphi_{0}\right)$ for the 0 -closure operator $\varphi_{0}$ defined by $\varphi_{0}(\mathbf{0})=\mathbf{0}$ and $\varphi_{0}(x)=\varphi(x)$ for $x>\mathbf{0}$.

A kernel operator on $L[12]$ or a dual closure operator on $L[5]$ is an operator $\gamma$ that is isotone, anti-extensive $(x \geq \gamma(x))$ and idempotent. A kernel system on $L$ or dual closure system on $L$ is a subset $S \subseteq L$ that is stable under the supremum operation (in particular for the empty supremum, $\mathbf{0} \in S$ ). The map $\gamma \mapsto \operatorname{lnv}(\gamma)$ gives an isomorphism between the poset of kernel operators and that of kernel systems.

Let $L$ and $M$ be two complete lattices, and consider $(\varepsilon, \delta): M \rightleftharpoons L$; we say that $(\varepsilon, \delta)$ is an adjunction [12] if

$$
\forall x \in L, \forall y \in M, \quad \delta(x) \leq y \Longleftrightarrow x \leq \varepsilon(y) .
$$

Then $\delta$ is called the lower adjoint of $\varepsilon$ and $\varepsilon$ is called the upper adjoint of $\delta$ [12]. It is well-known $[12,14]$ that $\delta$ is a complete join-morphism (it commutes with the supremum operation), $\varepsilon$ is a complete meet-morphism (it commutes with the infimum operation), $\delta \varepsilon \delta=\delta, \varepsilon \delta \varepsilon=\varepsilon, \varepsilon \delta$ is a closure operator and $\delta \varepsilon$ is a kernel (dual closure) operator

A well-known example of adjunction is given by Minkowski operations [14]. Let $E=\mathbb{R}^{d}$ or $E=\mathbb{Z}^{d}$. For every $p \in E$ and $X \subseteq E$, let $X_{p}=\{x+p \mid x \in X\}$ be the translate of $X$ by $p$. We define the Minkowski addition $\oplus$ by

$$
X \oplus B=\bigcup_{b \in B} X_{b}=\bigcup_{x \in X} B_{x}=\{x+b \mid x \in X, b \in B\},
$$

and the Minkowski subtraction $\ominus$ by

$$
X \ominus B=\bigcap_{b \in B} X_{-b}=\left\{p \in E \mid B_{p} \subseteq X\right\} .
$$

Then the two maps $\delta_{B}: \mathcal{P}(E) \rightarrow \mathcal{P}(E): X \mapsto X \oplus B$ and $\varepsilon_{B}: \mathcal{P}(E) \rightarrow \mathcal{P}(E)$ : $X \mapsto X \ominus B$ are called the dilation by $B$ and erosion by $B$ respectively [14]; they form an adjunction $\left(\varepsilon_{B}, \delta_{B}\right)$. Thus $\delta_{B} \varepsilon_{B}: X \mapsto(X \ominus B) \oplus B$ is a kernel operator and $\varepsilon_{B} \delta_{B}: X \mapsto(X \oplus B) \ominus B$ is a closure operator; here $(X \ominus B) \oplus B$ is the union of all translates of $B$ that are included in $X$, while $(X \oplus B) \ominus B$ is obtained by adding to $X$ all portions of its complement $X^{c}$ that cannot be covered by a translate of $\check{B}=\{-b \mid b \in B\}$; for a symmetrical $B$ we have $\check{B}=B$.

For sets and (partial) partitions, we adopt the terminology and notation of $[21,22,23]$. Let $E$ be a "space", whose elements are called "points"; in fact $E$ is an arbitrary set of size at least 2 , although in practical examples we will often consider the Euclidean space $\mathbb{R}^{d}$, the digital space $\mathbb{Z}^{d}$, or a bounded interval in such spaces. Points of $E$ will be written $p, q, r, \ldots$, subsets of $E$ will be designated by upper-case letters $A, B, \ldots, Y, Z$ (except the empty set $\emptyset$ ), and families of subsets of $E$ will be written $\mathcal{A}, \mathcal{B}, \ldots, \mathcal{Y}, \mathcal{Z}$. 
A partial partition of $E$ is a family $\pi$ of subsets of $E$ that are non-empty and mutually disjoint; an element of $\pi$ is called a block [19]; the union of all blocks of $\pi$ is the support of $\pi$, written $\operatorname{supp}(\pi)$. Partial partitions of $E$ will be written $\pi, \pi^{\prime}, \pi_{1}, \pi^{1}, \ldots$. A partition of $E$ is a partial partition whose support is $E$. Every partial partition is a partition of its support. Given a partial partition $\pi$ of $E$ and $p \in E$, let $\mathrm{Cl}_{\pi}(p)=\emptyset$ if $p \notin \operatorname{supp}(\pi)$, while for $p \in \operatorname{supp}(\pi), \mathrm{Cl}_{\pi}(p)$ is the unique block of $\pi$ to which $p$ belongs; then $\mathrm{Cl}_{\pi}(p)$ is called the class of $p$ in $\pi[20]$.

Write $\Pi(E)$ for the set of all partitions of $E$, and $\Pi^{*}(E)$ for the set of all partial partitions of $E$. Thus $\Pi^{*}(E)=\bigcup_{A \in \mathcal{P}(E)} \Pi(A)$. Families of partial partitions will be designated by upper-case Greek letters $\Gamma, \ldots, \Omega$. Write $\varnothing$ for the empty partial partition having no block. For $A \in \mathcal{P}(E)$, let $\mathbf{0}_{A}$ be the partition of $A$ into its singletons, and $\mathbf{1}_{A}$ the partition of $A$ into a single block (or no block if $A=\emptyset)$ :

$$
\mathbf{0}_{A}=\{\{p\} \mid p \in A\} \quad \text { and } \quad \mathbf{1}_{A}=\{A\} \backslash\{\emptyset\}= \begin{cases}\{A\} & \text { if } A \neq \emptyset \\ \varnothing & \text { if } A=\emptyset .\end{cases}
$$

The well-known refinement ordering on partitions [19] extends to partial partitions. Given $\pi_{1}, \pi_{2} \in \Pi^{*}(E)$, we say that $\pi_{1}$ is finer than $\pi_{2}$, or that $\pi_{2}$ is coarser than $\pi_{1}$, and write $\pi_{1} \leq \pi_{2}$ (or $\pi_{2} \geq \pi_{1}$ ), iff every block of $\pi_{1}$ is included in a block of $\pi_{2}$ :

$$
\pi_{1} \leq \pi_{2} \quad \Longleftrightarrow \quad \forall C_{1} \in \pi_{1}, \exists C_{2} \in \pi_{2}, C_{1} \subseteq C_{2} .
$$

Equivalently, point classes in $\pi_{1}$ are included in those of $\pi_{2}$ :

$$
\pi_{1} \leq \pi_{2} \quad \Longleftrightarrow \quad \forall p \in E, \mathrm{Cl}_{\pi_{1}}(p) \subseteq \mathrm{Cl}_{\pi_{2}}(p) .
$$

Then $\Pi^{*}(E)$, ordered by refinement, is a complete lattice with least element $\varnothing$ and greatest element $\mathbf{1}_{E}[8,20]$. Given a family $\left\{\pi_{i} \mid i \in I\right\}$ of partial partitions, the class map of their infimum $\bigwedge_{i \in I} \pi_{i}$ is given by intersection of the respective class maps:

$$
\forall p \in E, \quad \mathrm{Cl}_{\bigwedge_{i \in I} \pi_{i}}(p)=\bigcap_{i \in I} \mathrm{Cl}_{\pi_{i}}(p) .
$$

The class map of their supremum $\bigvee_{i \in I} \pi_{i}$ is given by chaining [19] class maps: for $p, q \in E, q \in \mathrm{Cl}_{\bigvee_{i \in I} \pi_{i}}(p)$ iff there is some integer $n \geq 1$ and a sequence $x_{0}, \ldots, x_{n}$ in $E$ with $x_{0}=p$ and $x_{n}=q$, such that for each $t=1, \ldots, n$ there is some $i(t) \in I$ with $x_{t} \in \mathrm{Cl}_{\pi_{i(t)}}\left(x_{t-1}\right)$.

For $A \in \mathcal{P}(E)$, the non-empty supremum and infimum operations in $\Pi^{*}(A)$ are inherited from $\Pi^{*}(E)$ : for a non-void $\left\{\pi_{i} \mid i \in I\right\} \subseteq \Pi^{*}(A), \bigvee_{i \in I} \pi_{i}$ and $\bigwedge_{i \in I} \pi_{i}$ are the same in $\Pi^{*}(A)$ and in $\Pi^{*}(E)$.

We have $\Pi(E)=\left\{\pi \in \Pi^{*}(E) \mid \pi \geq \mathbf{0}_{E}\right\}$. Now $\Pi(E)$, ordered by refinement, is a complete lattice with least element $0_{E}$ and greatest element $\mathbf{1}_{E}$, and whose and non-empty supremum and infimum operations are inherited from $\Pi^{*}(E)$. 


\section{Relating ClOSURE Systems ON SETS AND ON PARTiAl PARTITIONS}

This section extends some results from $[16,17]$. Note that [17] logically precedes [16], although it was published after the latter.

The fact that an infimum of partial partitions is obtained by intersecting their respective blocks, see (1), is at the basis of the relation between closure systems on $\mathcal{P}(E)$ and those on $\Pi^{*}(E)$. However, since intersecting blocks can give the empty set, we will have to consider 0 -closure systems on $\mathcal{P}(E)$, that is, closure systems $\mathcal{S}$ on $\mathcal{P}(E)$ such that $\emptyset \in \mathcal{S}$.

Given a family $\mathcal{S} \subseteq \mathcal{P}(E), \Pi(E) \cap \mathcal{P}(\mathcal{S} \backslash\{\emptyset\})$ and $\Pi^{*}(E) \cap \mathcal{P}(\mathcal{S} \backslash\{\emptyset\})$ will be the families respectively of partitions and of partial partitions, whose blocks belong to $\mathcal{S}$ (in fact, blocks are non-void, so they belong to $\mathcal{S} \backslash\{\emptyset\}$ ). Conversely, given a family $\Sigma \subseteq \Pi^{*}(E), \bigcup \Sigma$ will be the family of all blocks from all $\pi \in \Sigma$.

Let us write $\mathcal{P}_{0}(\mathcal{P}(E))$ for the set of all $\mathcal{S} \in \mathcal{P}(\mathcal{P}(E))$ such that $\emptyset \in \mathcal{S}$. Define the 4 maps

$$
\begin{aligned}
& \Delta \quad: \quad \mathcal{P}(\Pi(E)) \rightarrow \mathcal{P}_{0}(\mathcal{P}(E)) \quad: \quad \Sigma \mapsto\{\emptyset\} \cup(\bigcup \Sigma), \\
& \Delta^{*}: \mathcal{P}\left(\Pi^{*}(E)\right) \rightarrow \mathcal{P}_{0}(\mathcal{P}(E)) \quad: \quad \Sigma \mapsto\{\emptyset\} \cup(\bigcup \Sigma), \\
& \nabla \quad: \quad \mathcal{P}_{0}(\mathcal{P}(E)) \rightarrow \mathcal{P}(\Pi(E)) \quad: \quad \mathcal{S} \mapsto \Pi(E) \cap \mathcal{P}(\mathcal{S} \backslash\{\emptyset\}), \\
& \nabla^{*} \quad: \quad \mathcal{P}_{0}(\mathcal{P}(E)) \rightarrow \mathcal{P}\left(\Pi^{*}(E)\right) \quad: \quad \mathcal{S} \mapsto \Pi^{*}(E) \cap \mathcal{P}(\mathcal{S} \backslash\{\emptyset\}) .
\end{aligned}
$$

The two maps $\Delta$ and $\nabla$ coincide respectively with the maps $c$ and $e$ defined in [16] and [17] respectively. Then $\Delta^{*}$ and $\nabla^{*}$ are their extensions to partial partitions.

Lemma 1. $\left(\nabla^{*}, \Delta^{*}\right): \mathcal{P}_{0}(\mathcal{P}(E)) \rightleftharpoons \mathcal{P}\left(\Pi^{*}(E)\right)$ and $(\nabla, \Delta): \mathcal{P}_{0}(\mathcal{P}(E)) \rightleftharpoons$ $\mathcal{P}(\Pi(E))$ are adjunctions. For any $\left.\left.\mathcal{S} \in \mathcal{P}_{0}(\mathcal{P}(E)), \Delta(\nabla \mathcal{S})\right) \subseteq \mathcal{S}=\Delta^{*}\left(\nabla^{*} \mathcal{S}\right)\right)$.

Proof. Let $\Sigma \in \mathcal{P}\left(\Pi^{*}(E)\right)$ and $\mathcal{S} \in \mathcal{P}_{0}(\mathcal{P}(E))$. As $\Sigma \subseteq \Pi^{*}(E)$, we have

$$
\begin{aligned}
& \Sigma \subseteq \Pi^{*}(E) \cap \mathcal{P}(\mathcal{S} \backslash\{\emptyset\}) \Longleftrightarrow \Sigma \subseteq \mathcal{P}(\mathcal{S} \backslash\{\emptyset\}) \\
& \Longleftrightarrow \forall \pi \in \Sigma, \pi \subseteq \mathcal{S} \backslash\{\emptyset\} \Longleftrightarrow \bigcup \Sigma \subseteq \mathcal{S} \backslash\{\emptyset\},
\end{aligned}
$$

and as $\emptyset \in \mathcal{S}$, the latter is equivalent to $\{\emptyset\} \cup(\bigcup \Sigma) \subseteq \mathcal{S}$. Thus $\Delta^{*}(\Sigma) \subseteq \mathcal{S} \Longleftrightarrow$ $\Sigma \subseteq \nabla^{*}(\mathcal{S})$, so $\left(\nabla^{*}, \Delta^{*}\right)$ is an adjunction.

When $\Sigma \in \mathcal{P}(\Pi(E))$, we have $\Delta(\Sigma)=\Delta^{*}(\Sigma)$, and as $\Sigma \subseteq \Pi(E)$, we get $\Sigma \subseteq \nabla^{*}(\mathcal{S}) \Longleftrightarrow \Sigma \subseteq \nabla^{*}(\mathcal{S}) \cap \Pi(E)=\nabla(\mathcal{S})$; hence $\Delta(\Sigma) \subseteq \mathcal{S} \Longleftrightarrow \Sigma \subseteq \nabla(\mathcal{S})$, so $(\nabla, \Delta)$ is an adjunction.

From the two adjunctions $\left(\nabla^{*}, \Delta^{*}\right)$ and $(\nabla, \Delta)$ we get $\left.\Delta(\nabla \mathcal{S})\right) \subseteq \mathcal{S}$ and $\left.\Delta^{*}\left(\nabla^{*} \mathcal{S}\right)\right) \subseteq \mathcal{S}$. By definition, $\left.\emptyset \in \Delta^{*}\left(\nabla^{*} \mathcal{S}\right)\right)$; for $S \in \mathcal{S} \backslash\{\emptyset\}$, we have $\{S\} \in \Pi^{*}(E) \cap \mathcal{P}(\mathcal{S} \backslash\{\emptyset\})=\nabla^{*}(\mathcal{S})$, so $\left.S \in \bigcup \nabla^{*}(\mathcal{S}) \subseteq \Delta^{*}\left(\nabla^{*} \mathcal{S}\right)\right)$; hence $\left.\mathcal{S} \subseteq \Delta^{*}\left(\nabla^{*} \mathcal{S}\right)\right)$, and the equality follows.

We will now show how these two adjunctions link 0-closure systems on $\mathcal{P}(E)$ with closure systems on $\Pi^{*}(E)$ and on $\Pi(E)$, generalizing the analysis of $[16,17]$. We deal first with the case of partial partitions, as it is simpler. 
Theorem 2. For any $\mathcal{S} \in \mathcal{P}_{0}(\mathcal{P}(E))$, the following three statements are equivalent:

(1) $\mathcal{S}$ is a 0-closure system on $\mathcal{P}(E)$.

(2) $\nabla^{*}(\mathcal{S})$ is a closure system on $\Pi^{*}(E)$.

(3) There is a closure system $\Sigma$ on $\Pi^{*}(E)$ such that $\mathcal{S}=\Delta^{*}(\Sigma)$.

Proof. $1 \Rightarrow 2$. Let $\mathcal{S}$ be a 0 -closure system on $\mathcal{P}(E)$, and let $\Gamma \subseteq \nabla^{*}(\mathcal{S})$. If $\Gamma$ is empty, then $\bigwedge \Gamma=\mathbf{1}_{E}$, and as $E \in \mathcal{S}$ (since $\mathcal{S}$ is a closure system), $\mathbf{1}_{E} \in \nabla^{*}(\mathcal{S})$. Assume now $\Gamma$ non-empty. For any $\pi \in \Gamma$, all blocks of $\Gamma$ belong to $\mathcal{S} \backslash\{\emptyset\}$, hence for any $p \in E, \mathrm{Cl}_{\pi}(p) \in \mathcal{S}$. By (1) we have $\mathrm{Cl}_{\wedge \Gamma}(p)=\bigcap_{\pi \in \Gamma} \mathrm{Cl}_{\pi}(p)$, and as $\mathcal{S}$ is a closure system, this intersection belongs to $\mathcal{S}$. Hence all blocks of $\Lambda \Gamma$ belong to $\mathcal{S} \backslash\{\emptyset\}$, that is, $\Lambda \Gamma \in \nabla^{*}(\mathcal{S})$. Therefore $\nabla^{*}(\mathcal{S})$ is a closure system on $\Pi^{*}(E)$.

$2 \Rightarrow 3$. By Lemma $\left.1, \mathcal{S}=\Delta^{*}\left(\nabla^{*} \mathcal{S}\right)\right)$.

$3 \Rightarrow 1$. Let $\Sigma$ be a closure system on $\Pi^{*}(E)$ and let $\mathcal{C} \subseteq \Delta^{*}(\Sigma)$. If $\mathcal{C}$ is empty, then $\bigcap \mathcal{C}=E$, and as $\mathbf{1}_{E} \in \Sigma$ (since $\Sigma$ is a closure system), $E \in \Delta^{*}(\Sigma)$. Assume now $\mathcal{C}$ non-empty. By definition, $\emptyset \in \Delta^{*}(\Sigma)$. Thus if $\cap \mathcal{C}=\emptyset$, in particular if $\emptyset \in \mathcal{C}$, then $\bigcap \mathcal{C} \in \Delta^{*}(\Sigma)$. Suppose now that $\bigcap \mathcal{C} \neq \emptyset$, in particular $\emptyset \notin \mathcal{C}$; then for each $A \in \mathcal{C}$, let $\Sigma(A)=\{\pi \in \Sigma \mid A \in \pi\}$; since $A \in \bigcup \Sigma$, we have $\Sigma(A) \neq \emptyset$. Let $p \in \bigcap \mathcal{C}$; for any $A \in \mathcal{C}$ and $\pi \in \Sigma(A), \mathrm{Cl}_{\pi}(p)=A$. Let $\pi_{\mathcal{C}}=\bigwedge_{A \in \mathcal{C}} \bigwedge \Sigma(A)$; since $\Sigma$ is a closure system, $\pi_{\mathcal{C}} \in \Sigma$; then by (1) we have $\mathrm{Cl}_{\pi_{\mathcal{C}}}(p)=\bigcap \mathcal{C}$, so $\bigcap \mathcal{C}$ is a block of $\pi_{\mathcal{C}} \in \Sigma$, and $\bigcap \mathcal{C} \in \Delta^{*}(\Sigma)$. Therefore $\Delta^{*}(\Sigma)$ is a 0 -closure system.

Let us now exchange the roles of $\mathcal{P}(E)$ and $\Pi^{*}(E)$ :

Corollary 3. For any $\Sigma \in \mathcal{P}\left(\Pi^{*}(E)\right)$, consider the following three statements:

(1) $\Sigma$ is a closure system on $\Pi^{*}(E)$.

(2) $\Delta^{*}(\Sigma)$ is a 0 -closure system on $\mathcal{P}(E)$.

(3) There is a 0 -closure system $\mathcal{S}$ on $\mathcal{P}(E)$ such that $\Sigma=\nabla^{*}(\mathcal{S})$.

Then $3 \Rightarrow 1 \Rightarrow 2$. When there is some $\mathcal{C} \in \mathcal{P}_{0}(\mathcal{P}(E))$ such that $\Sigma=\nabla^{*}(\mathcal{C})$, the three statements are equivalent.

Proof. The implications $3 \Rightarrow 1$ and $1 \Rightarrow 2$ correspond to implications $1 \Rightarrow 2$ and $3 \Rightarrow 1$ respectively of Theorem 2 . The adjunction $\left(\nabla^{*}, \Delta^{*}\right)$ gives $\nabla^{*} \Delta^{*} \nabla^{*}=\nabla^{*}$; hence for $\Sigma=\nabla^{*}(\mathcal{C})$ we get $\nabla^{*}\left(\Delta^{*}(\Sigma)\right)=\nabla^{*}\left(\Delta^{*}\left(\nabla^{*}(\mathcal{C})\right)\right)=$ $\nabla^{*}(\mathcal{C})=\Sigma$, so $2 \Rightarrow 3$.

Since $\nabla^{*} \Delta^{*} \nabla^{*}=\nabla^{*}$, the condition "there is some $\mathcal{C} \in \mathcal{P}_{0}(\mathcal{P}(E))$ such that $\Sigma=\nabla^{*}(\mathcal{C})$ " is equivalent to $\nabla^{*}\left(\Delta^{*}(\Sigma)\right)=\Sigma$.

Let us now turn to the case of partitions. We need to describe a property introduced first in [17]. Let $\mathcal{S}$ be a 0 -closure system on $\mathcal{P}(E)$, and let $\varphi$ be the corresponding 0 -closure operator on $\mathcal{P}(E)$, i.e., $\mathcal{S}=\operatorname{lnv}(\varphi)$. For any $p \in E$, write $\varphi(p)$ for $\varphi(\{p\})$. Now $\mathcal{S}$, ordered by inclusion, is a complete lattice where 
the infimum is given by the intersection, and $\emptyset$ is the least element. For $A \in \mathcal{S}$ and $p \in A, \varphi(p) \subseteq A$ and $\varphi(p) \in \mathcal{S}$, thus $A$ is an atom of $\mathcal{S}$ iff for any $p \in A$, $\varphi(p)=A$. In particular, every atom is of the form $\varphi(p), p \in E$. However, for $p \in E, \varphi(p)$ is not necessarily an atom.

Lemma 4. Let $\varphi$ be a 0 -closure operator on $\mathcal{P}(E)$ and let $\mathcal{S}=\operatorname{Inv}(\varphi)$. Then the following three statements are equivalent:

(1) For every $p \in E, \varphi(p)$ is an atom of $\mathcal{S}$.

(2) The set of all $\varphi(p), p \in E$, is a partition of $E$.

(3) The set of all atoms of $\mathcal{S}$ is a partition of $E$.

Proof. $1 \Rightarrow 2$. The atoms of $\mathcal{S}$ are the $\varphi(p), p \in E$. Given $p, q \in E$ such that $\varphi(p) \neq \varphi(q), \varphi(p) \cap \varphi(q) \in \mathcal{S}$, and since $\varphi(p)$ and $\varphi(q)$ are atoms of $\mathcal{S}$, we get $\varphi(p) \cap \varphi(q)=\emptyset$. Thus the $\varphi(p), p \in E$, are non-void, pairwise disjoint, and cover $E$, i.e., they constitute a partition of $E$.

$2 \Rightarrow 3$. Let $p, q \in E$ such that $q \in \varphi(p)$; then $\varphi(q) \subseteq \varphi(p)$, and as $\varphi(p)$ and $\varphi(q)$ are blocks of a partition, we get $\varphi(q)=\varphi(p)$; this means that $\varphi(p)$ is an atom of $\mathcal{S}$. Hence the atoms of $\mathcal{S}$ are the $\varphi(p), p \in E$, and they constitute a partition of $E$.

$3 \Rightarrow 1$. For any $p \in E, p$ is covered by the partition of atoms of $\mathcal{S}$, so there is an atom $A$ with $p \in A$; then $\varphi(p)=A$, and $\varphi(p)$ is an atom of $\mathcal{S}$.

Note that the condition "there is a partition of $E$ whose blocks are of the form $\varphi(p), p \in E$ ", is not sufficient. In [17], condition 2 was called "condition P". Therefore we call a P-0-closure system a 0-closure system satisfying the properties of Lemma 4. Now this property can be linked to the adjunction $(\nabla, \Delta)$; the following is a slight generalization of Lemma 2.b of [17]:

Proposition 5. For any 0-closure system $\mathcal{S}$ on $\mathcal{P}(E), \Delta(\nabla(\mathcal{S}))$ is the greatest $P$-0-closure system included in $\mathcal{S}$, and $\nabla(\Delta(\nabla(\mathcal{S})))=\nabla(\mathcal{S})$. In particular $\mathcal{S}$ is a $P$-0-closure system iff $\Delta(\nabla(\mathcal{S}))=\mathcal{S}$.

Proof. We first prove that $\mathcal{S}$ is a P-0-closure system iff $\Delta(\nabla(\mathcal{S}))=\mathcal{S}$. Let $\mathcal{S}$ be a $\mathrm{P}$-0-closure system, and let $\mathcal{A}$ be the set of atoms of $\mathcal{S}$. Let $B \in \mathcal{S} \backslash\{\emptyset\}$; for $A \in \mathcal{A}$, we have either $A \subseteq B$ or $A \cap B=\emptyset$; since $\mathcal{A}$ constitutes a partition of $E$, this means that $\{A \in \mathcal{A} \mid A \cap B=\emptyset\}$ is a partition of $E \backslash B$. Hence $\pi_{B}=\{B\} \cup\{A \in \mathcal{A} \mid A \cap B=\emptyset\}$ is a partition with blocks in $\mathcal{S}$, that is, $\pi_{B} \in \nabla(\mathcal{S})$, and as $B \in \pi_{B}$, we get $B \in \bigcup \nabla(\mathcal{S}) \subseteq \Delta(\nabla(\mathcal{S}))$. Since $\emptyset \in \Delta(\nabla(\mathcal{S}))$, we deduce that $\mathcal{S} \subseteq \Delta(\nabla(\mathcal{S}))$. But $\Delta(\nabla \mathcal{S})) \subseteq \mathcal{S}$ by Lemma 1 , so the equality follows.

Let $\mathcal{S}$ be a 0 -closure system such that $\Delta(\nabla(\mathcal{S}))=\mathcal{S}$. Let $p, q \in E$ such that $q \in \varphi(p)$; then $\varphi(q) \subseteq \varphi(p)$. Now $\varphi(q) \in \Delta(\nabla(\mathcal{S}))$, that is, there is some $\pi \in \nabla(\mathcal{S})$ (a partition with blocks in $\mathcal{S}$ ) such that $\varphi(q) \in \pi$; there is some $B \in \pi$ such that $p \in B$, and as $B \in \mathcal{S}$, we get $\varphi(p) \subseteq B$; but $\varphi(q) \subseteq \varphi(p)$, 
with $\varphi(q), B \in \pi$, from which we deduce that $\varphi(q)=\varphi(p)=B$. Hence $\forall p \in E$, $q \in \varphi(p) \Rightarrow \varphi(q)=\varphi(p)$, so $\varphi(p)$ is an atom of $\mathcal{S}$.

Now we prove the general result. Let $\mathcal{S}$ be a 0 -closure system on $\mathcal{P}(E)$. From the adjunction $(\nabla, \Delta), \Delta \nabla$ is a kernel operator (dual closure), so $\Delta(\nabla(\mathcal{S})) \subseteq \mathcal{S}$ and $\Delta(\nabla(\Delta(\nabla(\mathcal{S}))))=\Delta(\nabla(\mathcal{S}))$, from which we deduce that $\Delta(\nabla(\mathcal{S}))$ is a P-0closure system. Let $\mathcal{T}$ be a P-0-closure system such that $\mathcal{T} \subseteq \mathcal{S}$; then $\Delta(\nabla(\mathcal{T}))=$ $\mathcal{T}$, and since $\Delta \nabla$ is isotone, $\Delta(\nabla(\mathcal{T})) \subseteq \Delta(\nabla(\mathcal{S})$ ); hence $\mathcal{T} \subseteq \Delta(\nabla(\mathcal{S}))$. Thus $\Delta(\nabla(\mathcal{S}))$ is the greatest $\mathrm{P}-0$-closure system included in $\mathcal{S}$.

We give now the analogue of Theorem 2 for partitions; its last sentence was proved in Theorem 1.a of [17].

Theorem 6. For any $\mathcal{S} \in \mathcal{P}_{0}(\mathcal{P}(E))$, the following three statements are equivalent:

(1) $\mathcal{S}$ is a P-0-closure system on $\mathcal{P}(E)$.

(2) $\nabla(\mathcal{S})$ is a closure system on $\Pi(E)$ and there is some $\Gamma \in \mathcal{P}(\Pi(E))$ such that $\mathcal{S}=\Delta(\Gamma)$.

(3) There is a closure system $\Sigma$ on $\Pi(E)$ such that $\mathcal{S}=\Delta(\Sigma)$.

Furthermore, for any 0-closure system $\mathcal{S}$ on $\mathcal{P}(E), \nabla(\mathcal{S})$ is a closure system on $\Pi(E)$.

Proof. $1 \Rightarrow 2$. By Theorem $2, \nabla^{*}(\mathcal{S})$ is a closure system on $\Pi^{*}(E)$. Now $\nabla(\mathcal{S})=\nabla^{*}(\mathcal{S}) \cap \Pi(E)$; since $\Pi(E)$ is a closure system on $\Pi^{*}(E)$, and the infimum operation in the complete lattice $\Pi(E)$ (including the empty infimum, i.e., greatest element) is inherited from $\Pi^{*}(E)$, it follows that $\nabla(\mathcal{S})$ is a closure system on $\Pi(E)$. By Proposition $5, \mathcal{S}=\Delta(\nabla(\mathcal{S}))$ with $\nabla(\mathcal{S}) \in \mathcal{P}(\Pi(E))$.

$2 \Rightarrow 3$. By the adjunction $(\nabla, \Delta), \Delta \nabla \Delta=\Delta$, so $\mathcal{S}=\Delta(\Gamma)=\Delta(\nabla(\Delta(\Gamma)))$ $=\Delta(\nabla(\mathcal{S}))$, where $\nabla(\mathcal{S})$ is a closure system on $\Pi(E)$.

$3 \Rightarrow 1$. Since the infimum operation in the complete lattice $\Pi(E)$ is inherited from $\Pi^{*}(E), \Sigma$ is a closure system on $\Pi^{*}(E)$, and $\Delta(\Sigma)=\Delta^{*}(\Sigma)$. By Theorem 2, $\mathcal{S}=\Delta^{*}(\Sigma)$ is a 0 -closure system on $\mathcal{P}(E)$. The adjunction $(\nabla, \Delta)$ gives $\Delta \nabla \Delta=\Delta$, hence $\Delta(\nabla(\mathcal{S}))=\Delta(\nabla(\Delta(\Sigma)))=\Delta(\Sigma)=\mathcal{S}$, so $\mathcal{S}$ is a P-0closure system by Proposition 5 .

Let $\mathcal{S}$ be 0 -closure system on $\mathcal{P}(E)$. By Proposition $5, \Delta(\nabla(\mathcal{S}))$ is a P-0closure system and $\nabla(\Delta(\nabla(\mathcal{S})))=\nabla(\mathcal{S})$, so $\nabla(\mathcal{S})$ is a closure system on $\Pi(E)$ (by $1 \Rightarrow 2$ ). Otherwise we remark that in the proof of $1 \Rightarrow 2$, property $\mathrm{P}$ was not used for showing that $\nabla(\mathcal{S})$ is a closure system.

In item 2, the condition "there is some $\Gamma \in \mathcal{P}(\Pi(E))$ such that $\mathcal{S}=\Delta(\Gamma)$ " is equivalent to $\Delta(\nabla(\mathcal{S}))=\mathcal{S}$, and to "for $\mathcal{T} \subset \mathcal{S}, \nabla(\mathcal{T}) \subset \nabla(\mathcal{S})$ ". Comparing Theorem 2 to Theorem 6 , we see that in the latter this condition "there is some $\Gamma \in \mathcal{P}(\Pi(E)$ ) such that $\mathcal{S}=\Delta(\Gamma)$ " has been added in item 2. Indeed, given $\mathcal{S} \in \mathcal{P}_{0}(\mathcal{P}(E))$ such that $\nabla(\mathcal{S})$ is a closure system on $\Pi(E), \mathcal{S}$ will not necessarily 
be a closure system on $\mathcal{P}(E)$. Let for example the set $E$ be the disjoint union of 4 non-void subsets $A, B, C, D$, and take $\mathcal{S}=\{\emptyset, A \cup B, A \cup C, E\}$; then $\mathcal{S}$ is not a closure system on $\mathcal{P}(E)$ because $(A \cup B) \cap(A \cup C)=A \notin \mathcal{S}$, but $\nabla(\mathcal{S})=\left\{\mathbf{1}_{E}\right\}$ is a closure system on $\Pi(E)$.

We obtain then the analogue of Corollary 3 for partitions:

Corollary 7. For any $\Sigma \in \mathcal{P}(\Pi(E))$, consider the following three statements:

(1) $\Sigma$ is a closure system on $\Pi(E)$.

(2) $\Delta(\Sigma)$ is a P-0-closure system on $\mathcal{P}(E)$.

(3) There is a P-0-closure system $\mathcal{S}$ on $\mathcal{P}(E)$ such that $\Sigma=\nabla(\mathcal{S})$.

Then $3 \Rightarrow 1 \Rightarrow 2$. When there is some $\mathcal{C} \in \mathcal{P}_{0}(\mathcal{P}(E))$ such that $\Sigma=\nabla(\mathcal{C})$, the three statements are equivalent.

Proof. The implications $3 \Rightarrow 1$ and $1 \Rightarrow 2$ follow from implications $1 \Rightarrow 2$ and $3 \Rightarrow 1$ respectively of Theorem 6 . The adjunction $(\nabla, \Delta)$ gives $\nabla \Delta \nabla=\nabla$; hence for $\Sigma=\nabla(\mathcal{C})$ we get $\nabla(\Delta(\Sigma))=\nabla(\Delta(\nabla(\mathcal{C})))=\nabla(\mathcal{C})=\Sigma$, so $2 \Rightarrow 3$.

Since $\nabla \Delta \nabla=\nabla$, the condition "there is some $\mathcal{C} \in \mathcal{P}_{0}(\mathcal{P}(E))$ such that $\Sigma=\nabla(\mathcal{C})$ " is equivalent to $\nabla(\Delta(\Sigma))=\Sigma$. In [17], the property $\nabla(\Delta(\Sigma))=\Sigma$ was called " $\Sigma$ is closed under reconstruction", and Proposition 1.e there showed that it leads to the equivalence between items 1 and 3 of Corollary 7 .

(a)

\begin{tabular}{|l|l|}
\hline$A$ & $B$ \\
\hline$C$ & $D$ \\
\hline
\end{tabular}

(c)

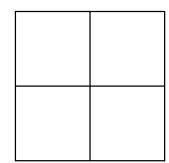

(b)
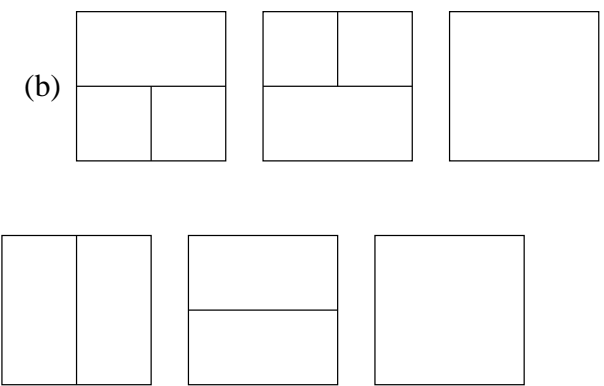
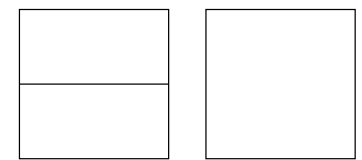

Figure 1. Here $E=\mathbb{Z}^{2}$. (a) The 4 sets $A, B, C, D$ partitioning $E$. (b) The set $\Sigma_{0}$ of 3 partitions satisfies item 2 , but not item 1 . (c) The set $\Sigma_{1}$ of 4 partitions satisfies item 1, but not item 3 .

Example 8. When we do not have $\Sigma=\nabla(\mathcal{C})$ for some $\mathcal{C} \in \mathcal{P}_{0}(\mathcal{P}(E))$, we will not obtain any other implication than $3 \Rightarrow 1 \Rightarrow 2$, as show the following two counter-examples. Let the set $E$ be the disjoint union of 4 non-void subsets $A, B, C, D$, see Figure 1(a).

(1) See Figure 1(b). Let

$$
\Sigma_{0}=\{\{A \cup B, C, D\},\{A, B, C \cup D\},\{E\}\} .
$$


Then

$$
\Delta\left(\Sigma_{0}\right)=\{\emptyset, A, B, C, D, A \cup B, C \cup D, E\}
$$

is a P-0-closure system on $\Pi(E)$, but $\Sigma_{0}$ is not a closure system on $\Pi(E)$, because $\bigwedge \Sigma_{0}=\{A, B, C, D\} \notin \Sigma_{0}$. Thus $\Sigma_{0}$ satisfies item 2, but not item 1.

(2) See Figure 1(c). Let

$$
\Sigma_{1}=\{\{A, B, C, D\},\{A \cup C, B \cup D\},\{A \cup B, C \cup D\},\{E\}\} .
$$

Then $\Sigma_{1}$ is a closure system on $\Pi(E)$; since $\{A \cup B, C, D\} \in \nabla\left(\Delta\left(\Sigma_{1}\right)\right) \backslash$ $\Sigma_{1}, \nabla\left(\Delta\left(\Sigma_{1}\right)\right) \neq \Sigma_{1}$, hence we cannot have $\mathcal{S} \in \mathcal{P}_{0}(\mathcal{P}(E))$ with $\Sigma_{1}=$ $\nabla(\mathcal{S})$. Thus $\Sigma_{1}$ satisfies item 1 , but not item 3 .

These two counter-examples remain valid with $\Pi^{*}(E), \nabla^{*}$ and $\Delta^{*}$ instead of $\Pi(E), \nabla$ and $\Delta$. So in Corollary 3 too, we will not have any other implication than $3 \Rightarrow 1 \Rightarrow 2$ when we do not have $\Sigma=\nabla^{*}(\mathcal{C})$ for some $\mathcal{C} \in \mathcal{P}_{0}(\mathcal{P}(E))$.

\section{Closures from operators aCting on Blocks}

Given a 0 -closure system $\mathcal{S}$ on $\mathcal{P}(E), \nabla^{*}(\mathcal{S})$ is a closure system on $\Pi^{*}(E)$; we will now describe how to construct the closure operator on $\Pi^{*}(E)$ corresponding to $\nabla^{*}(\mathcal{S})$ from the 0 -closure operator on $\mathcal{P}(E)$ corresponding to $\mathcal{S}$. In particular, we will see that under some conditions it can be achieved by taking the limit of the iterated application of some specific set operators on the blocks of a partial partition.

Let $\varphi$ be a 0 -closure operator on $\mathcal{P}(E)$. Since $\operatorname{lnv}(\varphi)$ is a 0 -closure system on $\mathcal{P}(E), \nabla^{*}(\operatorname{Inv}(\varphi))=\Pi^{*}(E) \cap \mathcal{P}(\operatorname{Inv}(\varphi) \backslash\{\emptyset\})$, the set of partial partitions with blocks in $\operatorname{lnv}(\varphi)$, is by Theorem 2 a closure system on $\Pi^{*}(E)$. There is thus a closure operator $\mathrm{C}(\varphi)$ on $\Pi^{*}(E)$ such that $\operatorname{Inv}(\mathrm{C}(\varphi))=\nabla^{*}(\operatorname{Inv}(\varphi))$. For any $\pi \in \Pi^{*}(E), \mathrm{C}(\varphi)(\pi)$ is the least $\pi^{\prime} \in \Pi^{*}(E)$ such that $\pi^{\prime} \geq \pi$ and all blocks of $\pi^{\prime}$ are invariant under $\varphi$. Note that $\mathrm{C}(\varphi)(\emptyset)=\varnothing$, so $\mathrm{C}(\varphi)$ is a 0-closure operator. In [17], the restriction of $\mathrm{C}(\varphi)$ to $\Pi(E)$ was written $C_{e}$.

Following [21], given an operator $\psi$ on $\mathcal{P}(E)$, we define the blockwise extension of $\psi$ as the operator $\mathrm{B}(\psi)$ on $\Pi^{*}(E)$ given by

$$
\forall \pi \in \Pi^{*}(E), \quad \mathrm{B}(\psi)(\pi)=\bigvee_{B \in \pi} \mathbf{1}_{\psi(B)} .
$$

In other words, $\mathrm{B}(\psi)(\pi)$ is obtained by applying $\psi$ to each block of $\pi$, then recursively merging all overlapping blocks $\psi(B) \neq \emptyset, B \in \pi$. Usually one assumes that $\psi(\emptyset)=\emptyset$. Indeed, if $\psi$ is isotone and $\psi(\emptyset) \neq \emptyset$, then for any $\pi \in \Pi^{*}(E)$, $\mathrm{B}(\psi)(\pi)$ will have a single block (since all $\psi(B), B \in \pi$, overlap through $\psi(\emptyset)$ ); furthermore, defining $\psi^{\prime}$ by $\psi^{\prime}(\emptyset)=\emptyset$ and $\psi^{\prime}(X)=X$ for $X \in \mathcal{P}(E) \backslash\{\emptyset\}$, then $\psi^{\prime}$ inherits the isotony of $\psi$ and $\mathrm{B}\left(\psi^{\prime}\right)=\mathrm{B}(\psi)$. 
We recall a known fact $[14,26]$ : given an extensive isotone operator $\theta$ on a complete lattice $L$, then $\operatorname{Inv}(\theta)$ is a closure system on $L$, there is a unique closure operator $\widehat{\theta}$ on $L$ such that $\operatorname{lnv}(\hat{\theta})=\operatorname{lnv}(\theta)$, and $\widehat{\theta}$ is the least closure operator on $L$ which is $\geq \theta$. We get then the following consequence:

Proposition 9. Let $\psi$ be an extensive isotone operator on $\mathcal{P}(E)$ such that $\psi(\emptyset)=\emptyset$. Let $\varphi$ be the least closure operator on $\mathcal{P}(E)$ such that $\varphi \geq \psi$. Then $\mathrm{B}(\psi)$ is extensive and isotone, $\varphi$ is a 0-closure operator, and $\mathrm{C}(\varphi)$ is the least closure operator on $\Pi^{*}(E)$ such that $\mathrm{C}(\varphi) \geq \mathrm{B}(\psi)$.

Proof. Since $\psi(\emptyset)=\emptyset, \operatorname{lnv}(\psi)$ is a 0 -closure system on $\mathcal{P}(E)$, and as $\operatorname{lnv}(\varphi)=$ $\operatorname{lnv}(\psi), \varphi$ is a 0 -closure operator. By $[21] \mathrm{B}(\psi)$ inherits from $\psi$ the property of being extensive and isotone. Now $\operatorname{Inv}(\mathrm{C}(\varphi))=\nabla^{*}(\operatorname{Inv}(\varphi))$, the set of partial partitions with blocks in $\operatorname{Inv}(\varphi)=\operatorname{Inv}(\psi)$. For $\pi \in \nabla^{*}(\operatorname{Inv}(\varphi))$, each block $B \in \pi$ belongs to $\operatorname{lnv}(\varphi)=\operatorname{lnv}(\psi)$, that is, $\psi(B)=B$; by $(3)$, we get $\mathrm{B}(\psi)(\pi)=\pi$, thus $\pi \in \operatorname{Inv}(\mathrm{B}(\psi))$. For $\pi \notin \nabla^{*}(\operatorname{Inv}(\varphi))$, there is a block $B \in \pi$ that does not belong to $\operatorname{lnv}(\varphi)=\operatorname{lnv}(\psi)$, that is, $\psi(B) \neq B$; as $\psi$ is extensive, $B \subset \psi(B)$, and by (3) $\mathbf{1}_{\psi(B)} \leq \mathrm{B}(\psi)(\pi)$, in other words $\psi(B)$ is included in a block of $\mathrm{B}(\psi)(\pi)$; it follows then that for $p \in B$ we have $\mathrm{Cl}_{\pi}(p)=B \subset \psi(B) \subseteq \mathrm{Cl}_{\mathrm{B}(\psi)(\pi)}(p)$, in other words $\pi \neq \mathrm{B}(\psi)(\pi)$, thus $\pi \notin \operatorname{lnv}(\mathrm{B}(\psi))$. Therefore $\operatorname{Inv}(\mathrm{C}(\varphi))=\nabla^{*}(\operatorname{lnv}(\varphi))=$ $\operatorname{lnv}(\mathrm{B}(\psi))$, and as $\mathrm{C}(\varphi)$ is a closure operator, it must be the least one $\geq \mathrm{B}(\psi)$.

Given a 0 -closure operator $\varphi$ on $\mathcal{P}(E)$, for any $\pi \in \Pi^{*}(E)$ we will have $\mathrm{B}(\varphi)(\pi) \leq \mathrm{C}(\varphi)(\pi)$, but generally not the equality. This is for example the case for $E=\mathbb{Z}^{d}$ with the closure operator $\varphi=\varepsilon_{B} \delta_{B}: X \mapsto(X \oplus B) \ominus B$, the composition of the dilation and erosion by some bounded $B \in \mathcal{P}(E)$; as seen in Figure 2, for a finite partial partition, several iterations of $B(\varphi)$ may be necessary in order to reach a partial partition with blocks invariant under $\varphi$.

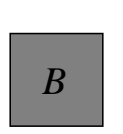

(a)

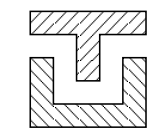

(b)

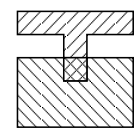

(c)

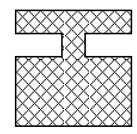

(d)

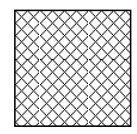

(e)

Figure 2. Here $E=\mathbb{Z}^{2}$. (a) The symmetrical bounded set $B$ (centered about the origin); we consider the closure operator $\varphi=\varepsilon_{B} \delta_{B}: X \mapsto(X \oplus B) \ominus B$. (b) A partial partition $\pi$ with 2 blocks $A_{1}, A_{2}$ (identified by their hatching). (c) Applying $\varphi$ to the blocks $A_{1}, A_{2}$, the two closed blocks $\varphi\left(A_{1}\right), \varphi\left(A_{2}\right)$ overlap. (d) $\mathrm{B}(\varphi)(\pi)$ has one block $\varphi\left(A_{1}\right) \cup \varphi\left(A_{2}\right)$. (e) $\mathrm{B}(\varphi)^{2}(\pi)$ has one block closed under $\varphi$, hence $\mathrm{B}(\varphi)^{2}(\pi)=\mathrm{C}(\varphi)(\pi)$. 
Proposition 10. Given a 0-closure operator $\varphi$ on $\mathcal{P}(E)$, for any finite $\pi \in$ $\Pi^{*}(E)$, there is some $n \in \mathbb{N}$ such that $\mathrm{B}(\varphi)^{n}(\pi)=\mathrm{C}(\varphi)(\pi)$.

Proof. For a finite partial partition $\pi=\left\{B_{1}, \ldots, B_{m}\right\}, \mathrm{B}(\varphi)(\pi)$ is the least partial partition such that each one of $\varphi\left(B_{1}\right), \ldots, \varphi\left(B_{m}\right)$ is included in one (and only one) of its blocks, and in particular each block of $\mathrm{B}(\varphi)(\pi)$ contains at least one of $\varphi\left(B_{1}\right), \ldots, \varphi\left(B_{m}\right)$; since $\varphi$ is extensive, $\varphi\left(B_{1}\right), \ldots, \varphi\left(B_{m}\right)$ are all nonempty. Thus either $\varphi\left(B_{1}\right), \ldots, \varphi\left(B_{m}\right)$ are pairwise disjoint and constitute the $m$ blocks of $\mathrm{B}(\varphi)(\pi)$, or we have some $i, j(1 \leq i<j \leq m)$ with $\varphi\left(B_{i}\right) \cap \varphi\left(B_{j}\right) \neq \emptyset$, hence $\varphi\left(B_{i}\right) \cup \varphi\left(B_{j}\right)$ is included in the same block of $\mathrm{B}(\varphi)(\pi)$, and the latter has less than $m$ blocks. Therefore repeated application of $\mathrm{B}(\varphi)$ can only decrease the number of blocks, and there is thus some $t \in \mathbb{N}$ such that $\mathrm{B}(\varphi)^{t}(\pi)$ has the same number of blocks as $\mathrm{B}(\varphi)^{t+1}(\pi)$. This means that for $\mathrm{B}(\varphi)^{t}(\pi)=\left\{C_{1}, \ldots, C_{k}\right\}$ we have $\mathrm{B}(\varphi)^{t+1}(\pi)=\left\{\varphi\left(C_{1}\right), \ldots, \varphi\left(C_{k}\right)\right\}$, where $\varphi\left(C_{1}\right), \ldots, \varphi\left(C_{k}\right) \in \operatorname{lnv}(\varphi)$. Hence $\pi \leq \mathrm{B}(\varphi)^{t+1}(\pi) \in \operatorname{lnv}(\mathrm{C}(\varphi))$, where $\mathrm{C}(\varphi)$ is the least closure operator $\geq \mathrm{B}(\varphi)$ (thus $\left.\geq \mathrm{B}(\varphi)^{t+1}\right)$, we deduce then that $\mathrm{B}(\varphi)^{t+1}(\pi)=\mathrm{C}(\varphi)(\pi)$.

We show in Figure 3 an example of an infinite partial partition $\pi$ such that for the same closure operator $\varphi$ as in Figure 2, the sequence of $\mathrm{B}(\varphi)^{n}(\pi)$ is strictly increasing, and here $\mathrm{C}(\varphi)(\pi)=\bigvee_{n=1}^{\infty} \mathrm{B}(\varphi)^{n}(\pi)$.

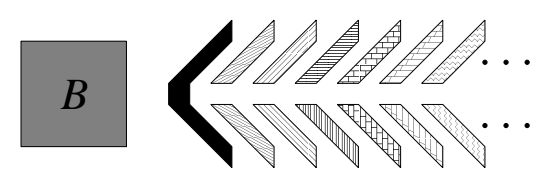

(a) (b)

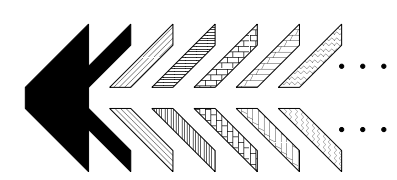

(c)

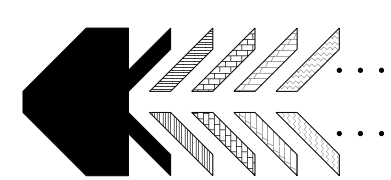

(d)

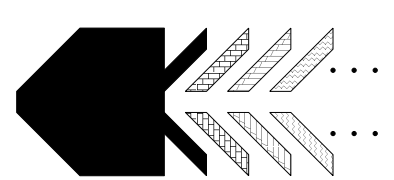

(e)

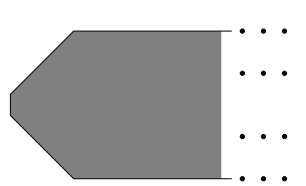

(f)

Figure 3. Here $E=\mathbb{Z}^{2}$. (a) The symmetrical bounded set $B$ (centered about the origin); we consider the closure operator $\varphi=\varepsilon_{B} \delta_{B}: X \mapsto(X \oplus B) \ominus B$. In the following partial partitions, each block is identified by its hatching or grey-level; blocks not invariant under $\varphi$ are shown in black (it will be the leftmost block in each). (b) An infinite partial partition $\pi$. (c) $\mathrm{B}(\varphi)(\pi)$. (d) $\mathrm{B}(\varphi)^{2}(\pi)$. (e) $\mathrm{B}(\varphi)^{3}(\pi)$. For any $n \in \mathbb{N}, \mathrm{B}(\varphi)^{n}(\pi)$ will always have its leftmost block not in $\operatorname{lnv}(\varphi)$. (f) $\bigvee_{n=1}^{\infty} \mathrm{B}(\varphi)^{n}(\pi)$ consists of one block in $\operatorname{lnv}(\varphi)$, so $\mathrm{C}(\varphi)(\pi)=\bigvee_{n=1}^{\infty} \mathrm{B}(\varphi)^{n}(\pi)$. 
This example suggests a form of semi-continuity of the operator. Given a nonempty directed set $D$ and a complete lattice $L$, for any isotone map $\lambda: D \rightarrow L$, the set $\{\lambda(d) \mid d \in D\}$ is directed; now an isotone operator $\theta$ on $L$ will be called $D \uparrow$-continuous if for any isotone map $\lambda: D \rightarrow L$ we have $\theta\left(\bigvee_{d \in D} \lambda(d)\right)=$ $\bigvee_{d \in D} \theta(\lambda(d))$. An interesting particular case is when $D=\mathbb{N}$ with the numerical order, and then following [15] we say that $\theta$ is $\uparrow$-continuous, and this means that for a sequence $x_{n} \in L, n \in \mathbb{N}$, that is monotonous increasing $(\forall n \in \mathbb{N}$, $\left.x_{n} \leq x_{n+1}\right)$, we have $\theta\left(\bigvee_{n \in \mathbb{N}} x_{n}\right)=\bigvee_{n \in \mathbb{N}} \theta\left(x_{n}\right)$

Proposition 11. Let $D$ be a non-empty directed set and let $\psi$ be a $D \uparrow$-continuous isotone operator on $\mathcal{P}(E)$. Then $\mathrm{B}(\psi)$ is $D \uparrow$-continuous and isotone.

Proof. By [21], $\mathrm{B}(\psi)$ inherits the isotony of $\psi$. Consider an isotone map $\lambda: D \rightarrow$ $\Pi^{*}(E)$. Since $\mathrm{B}(\psi)$ is isotone, we have $\mathrm{B}(\psi)\left(\bigvee_{d \in D} \lambda(d)\right) \geq \bigvee_{d \in D} \mathrm{~B}(\psi)(\lambda(d))$. Consider a block $B \in \bigvee_{d \in D} \lambda(d)$ and take $p \in B$; thus $B=\mathrm{Cl}_{\bigvee_{d \in D} \lambda(d)}(p)$. Since $\lambda$ and the map $\Pi^{*}(E) \rightarrow \mathcal{P}(E): \pi \mapsto \mathrm{Cl}_{\pi}(p)$ are isotone, the map $D \rightarrow$ $\mathcal{P}(E): \lambda \mapsto \mathrm{Cl}_{\lambda(d)}(p)$ will be isotone. Thus the set $\left\{\mathrm{Cl}_{\lambda(d)}(p) \mid d \in D\right\}$ is directed, so by [20] we have $B=\mathrm{Cl}_{\bigvee_{d \in D} \lambda(d)}(p)=\bigcup_{d \in D} \mathrm{Cl}_{\lambda(d)}(p)$. As $\psi$ is $D \uparrow$ continuous, $\psi(B)=\bigcup_{d \in D} \psi\left(\mathrm{Cl}_{\lambda(d)}(p)\right)$. Let $D_{p}=\left\{d \in D \mid \mathrm{Cl}_{\lambda(d)}(p) \neq \emptyset\right\}$; then $B=\bigcup_{d \in D_{p}} \mathrm{Cl}_{\lambda(d)}(p)$, so $D_{p} \neq \emptyset$. For $d \in D_{p}$ and $d^{\prime} \in D \backslash D_{p}, \mathrm{Cl}_{\lambda\left(d^{\prime}\right)}(p)=$ $\emptyset \subseteq \mathrm{Cl}_{\lambda(d)}(p)$, and as $\psi$ is isotone, $\psi\left(\mathrm{Cl}_{\lambda\left(d^{\prime}\right)}(p)\right) \subseteq \psi\left(\mathrm{Cl}_{\lambda(d)}(p)\right)$; hence $\psi(B)=$ $\bigcup_{d \in D_{p}} \psi\left(\mathrm{Cl}_{\lambda(d)}(p)\right)$. Thus there exists $d_{0} \in D_{p}$ such that $p \in \psi\left(\mathrm{Cl}_{\lambda\left(d_{0}\right)}(p)\right)$ and for any $q \in \psi(B)$, there exists $d_{1} \in D_{p}$ such that $q \in \psi\left(\mathrm{Cl}_{\lambda\left(d_{1}\right)}(p)\right)$; as $D$ is directed, there is some $d_{2} \in D$ with $d_{0}, d_{1} \leq d_{2}$, so $\psi\left(\mathrm{Cl}_{\lambda\left(d_{0}\right)}(p)\right), \psi\left(\mathrm{Cl}_{\lambda\left(d_{1}\right)}(p)\right) \subseteq$ $\psi\left(\mathrm{Cl}_{\lambda\left(d_{2}\right)}(p)\right)$, hence $p, q \in \psi\left(\mathrm{Cl}_{\lambda\left(d_{2}\right)}(p)\right)$; now $\emptyset \subset \mathrm{Cl}_{\lambda\left(d_{0}\right)}(p) \subseteq \mathrm{Cl}_{\lambda\left(d_{2}\right)}(p)$, so $d_{2} \in D_{p}$. In other words all points of $\psi(B)$ are chained by the $\psi\left(\mathrm{Cl}_{\lambda(d)}(p)\right)$, $d \in D_{p}$. We deduce that $\bigvee_{d \in D_{p}} \mathbf{1}_{\psi\left(\mathrm{Cl}_{\lambda(d)}(p)\right)}=\mathbf{1}_{\psi(B)}$. Now for every $d \in D_{p}$, $\mathrm{Cl}_{\lambda(d)}(p)$ is the block of $\lambda(d)$ containing $p$; hence

$$
\begin{gathered}
\mathbf{1}_{\psi(B)}=\bigvee_{d \in D_{p}} \mathbf{1}_{\psi\left(\mathrm{Cl}_{\lambda(d)}(p)\right)} \leq \bigvee\left\{\mathbf{1}_{\psi(A)} \mid \exists d \in D, A \in \lambda(d)\right\} \\
=\bigvee_{d \in D} \bigvee_{A \in \lambda(d)} \mathbf{1}_{\psi(A)}=\bigvee_{d \in D} \mathrm{~B}(\psi)(\lambda(d)) .
\end{gathered}
$$

As this holds for any $B \in \bigvee_{d \in D} \lambda(d)$, we get

$$
\mathrm{B}(\psi)\left(\bigvee_{d \in D} \lambda(d)\right)=\bigvee\left\{\mathbf{1}_{\psi(B)} \mid B \in \bigvee_{d \in D} \lambda(d)\right\} \leq \bigvee_{d \in D} \mathrm{~B}(\psi)(\lambda(d))
$$

But $\mathrm{B}(\psi)\left(\bigvee_{d \in D} \lambda(d)\right) \geq \bigvee_{d \in D} \mathrm{~B}(\psi)(\lambda(d))$; hence the equality follows. Therefore $\mathrm{B}(\psi)$ is $D \uparrow$-continuous. 
Given an $\uparrow$-continuous isotone operator $\theta$ on a complete lattice $L, \bigvee_{n \in \mathbb{N}} \theta^{n}$ is the least closure operator on $L$ which is $\geq \theta$, and it is $\uparrow$-continuous [15]. We deduce then the following:

Corollary 12. Let $\psi$ be a $\uparrow$-continuous extensive isotone operator on $\mathcal{P}(E)$ such that $\psi(\emptyset)=\emptyset$, and let $\varphi$ be the least 0-closure operator on $\mathcal{P}(E)$ such that $\varphi \geq \psi$. Then $\mathrm{C}(\varphi)=\bigvee_{n \in \mathbb{N}} \mathrm{B}(\psi)^{n}$, and the closure operator $\mathrm{C}(\varphi)$ is $\uparrow$-continuous.

An isotone operator $\psi$ on $\mathcal{P}(E)$ is finitary if for all $X \in \mathcal{P}(E), \psi(X)=$ $\bigcup\{\psi(Y)|Y \in \mathcal{P}(E)| Y \mid,<\infty\}[3]$. Obviously every finitary operator is $\uparrow$ continuous, because for any increasing sequence of sets $X_{n}, n \in \mathbb{N}$, a finite subset of $\bigcup_{n \in \mathbb{N}} X_{n}$ is necessarily included in some $X_{n}$. For example for $E=\mathbb{R}^{d}$ or $E=\mathbb{Z}^{d}$, given a finite subset $B$ of $E$, the maps $\delta_{B}: X \mapsto X \oplus B$ and $\varepsilon_{B}: X \mapsto X \ominus B$, as well as their compositions $\delta_{B} \varepsilon_{B}$ and $\varepsilon_{B} \delta_{B}$ are finitary. This explains the convergence of $\mathrm{B}(\varphi)^{n}(\pi)$ to $\mathrm{C}(\varphi)(\pi)$ in Figure 3. Also in $\mathbb{R}^{d}$, the convex hull operator is finitary.

When $\psi$ is not $\uparrow$-continuous, $\mathrm{C}(\varphi)$ can be constructed as the limit of a transfinite iteration of $\mathrm{B}(\psi)$ : for a limit ordinal $\alpha$, set $\mathrm{B}(\psi)^{\alpha}(\pi)=\bigvee_{\beta<\alpha} \mathrm{B}(\psi)^{\beta}(\pi)$, and for the successor $\alpha+1$ of an ordinal $\alpha$, set $\mathrm{B}(\psi)^{\alpha+1}(\pi)=\mathrm{B}(\psi)\left(\mathrm{B}(\psi)^{\alpha}(\pi)\right)$; then for some ordinal $\alpha$ we obtain $\mathrm{B}(\psi)^{\alpha}=\mathrm{C}(\varphi)$. This construction was given in [17], with $\mathrm{B}(\varphi)$ in place of $\mathrm{B}(\psi)$, for the restriction of $\mathrm{C}(\varphi)$ to $\Pi(E)$.

Given an adjunction $(\varepsilon, \delta)$ on $\mathcal{P}(E)$, we say that $(\varepsilon, \delta)$ is regular $[20,21]$ if $\varepsilon(\emptyset)=\emptyset$, equivalently, $\forall X \in \mathcal{P}(E), X \neq \emptyset \Rightarrow \delta(X) \neq \emptyset$ (we have $\delta(\emptyset)=\emptyset$ anyway). In [21] we showed that for any regular adjunction $(\varepsilon, \delta)$ on $\mathcal{P}(E)$, $(\mathrm{B}(\varepsilon), \mathrm{B}(\delta))$ is an adjunction on $\Pi^{*}(E)$ and for any $\pi \in \Pi^{*}(E)$ we have $\mathrm{B}(\varepsilon)(\pi)=$ $\{\varepsilon(B) \mid B \in \pi, \varepsilon(B) \neq \emptyset\}$. Thus $\mathrm{B}(\varepsilon) \mathrm{B}(\delta)$ is a closure operator on $\Pi^{*}(E)$, and we can compare it to $\mathrm{C}(\varepsilon \delta)$ :

Proposition 13. Let $(\varepsilon, \delta)$ be a regular adjunction on $\mathcal{P}(E)$. Then $\mathrm{B}(\varepsilon) \mathrm{B}(\delta) \geq$ $\mathrm{C}(\varepsilon \delta)$.

Proof. Let $\pi \in \Pi^{*}(E)$ and let $\pi^{\prime}=\mathrm{B}(\delta)(\pi)$. Then $\mathrm{B}(\varepsilon) \mathrm{B}(\delta)(\pi)=\mathrm{B}(\varepsilon)\left(\pi^{\prime}\right)=$ $\left\{\varepsilon(B) \mid B \in \pi^{\prime}, \varepsilon(B) \neq \emptyset\right\}$. Now by the adjunction $(\varepsilon, \delta)$, for any $B \in \pi^{\prime}$ we have $\varepsilon \delta(\varepsilon(B))=\varepsilon(B)$, in other words all blocks of $\mathrm{B}(\varepsilon) \mathrm{B}(\delta)(\pi)=\mathrm{B}(\varepsilon)\left(\pi^{\prime}\right)$ belong to $\operatorname{lnv}(\varepsilon \delta)$, thus $\mathrm{B}(\varepsilon) \mathrm{B}(\delta)(\pi) \in \operatorname{lnv}(\mathrm{C}(\varepsilon \delta))$. Hence $\operatorname{lnv}(\mathrm{B}(\varepsilon) \mathrm{B}(\delta)) \subseteq \operatorname{lnv}(\mathrm{C}(\varepsilon \delta))$, and as $\mathrm{C}(\varepsilon \delta)$ and $\mathrm{B}(\varepsilon) \mathrm{B}(\delta)$ are closure operators, this means that $\mathrm{B}(\varepsilon) \mathrm{B}(\delta) \geq$ $\mathrm{C}(\varepsilon \delta)$.

For a partial partition $\pi$ with several blocks, we often have $\mathrm{B}(\varepsilon) \mathrm{B}(\delta)(\pi)>$ $\mathrm{C}(\varepsilon \delta)(\pi)$. Indeed, given two block $A_{1}, A_{2} \in \operatorname{lnv}(\varepsilon \delta)$, we can have $\delta\left(A_{1}\right) \cap \delta\left(A_{2}\right) \neq$ $\emptyset$, so $\mathrm{B}(\delta)\left(\left\{A_{1}, A_{2}\right\}\right)=\left\{\delta\left(A_{1}\right) \cup \delta\left(A_{2}\right)\right\}$ and $\mathrm{B}(\varepsilon) \mathrm{B}(\delta)\left(\left\{A_{1}, A_{2}\right\}\right)=\left\{\varepsilon\left(\delta\left(A_{1}\right) \cup\right.\right.$ $\left.\left.\delta\left(A_{2}\right)\right)\right\}$. See Figure 4 .

Given a closure operator $\Phi$ on $\Pi^{*}(E)$, the restriction of $\Phi$ to $\Pi(E)$ will be a closure operator on $\Pi(E)$. However [21] gives another construction: given a 


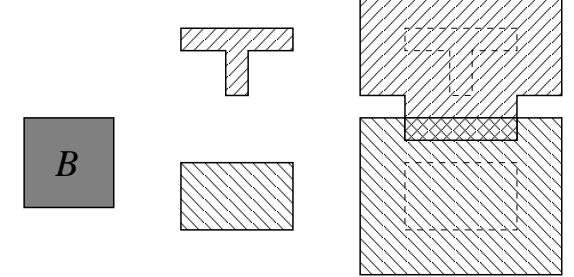

(a)

(b)

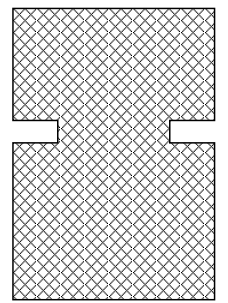

(d)

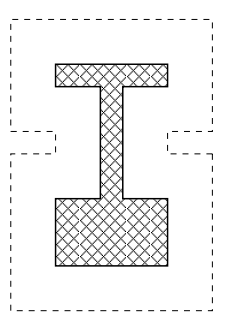

(e)

Figure 4. Here $E=\mathbb{Z}^{2}$. (a) The symmetrical bounded set $B$ (centered about the origin); we consider the dilation $\delta_{B}: X \mapsto X \oplus B$ and the erosion $\varepsilon_{B}: X \mapsto X \ominus B$. (b) A partial partition $\pi$ with 2 blocks $A_{1}, A_{2}$ (identified by their hatching), both invariant under the closure operator $\varepsilon_{B} \delta_{B}$. (c) Applying $\delta_{B}$ to the blocks $A_{1}, A_{2}$, the two dilated blocks $\delta_{B}\left(A_{1}\right), \delta_{B}\left(A_{2}\right)$ overlap. (d) $\mathrm{B}\left(\delta_{B}\right)(\pi)$ has one block $\delta_{B}\left(A_{1}\right) \cup \delta_{B}\left(A_{2}\right)$. (e) $\mathrm{B}\left(\varepsilon_{B}\right) \mathrm{B}\left(\delta_{B}\right)(\pi)$ has one block $\varepsilon_{B}\left(\delta_{B}\left(A_{1}\right) \cup \delta_{B}\left(A_{2}\right)\right)$, which is invariant under $\varepsilon_{B} \delta_{B}$.

partition $\pi$, first remove all singleton blocks of $\pi$, next apply $\Phi$ to the resulting partial partition, finally add all singleton blocks outside the support; in other words, we get the partition $\Phi^{\prime}(\pi)=\Phi\left(\pi \backslash \mathbf{0}_{E}\right) \vee \mathbf{0}_{E}$. Then $\Phi^{\prime}$ is a closure operator on $\Pi(E)$; moreover, if $\Phi$ is a 0 -closure operator on $\Pi^{*}(E)(\Phi(\varnothing)=\varnothing)$, then $\Phi^{\prime}$ will be a 0 -closure operator on $\Pi(E)\left(\Phi^{\prime}\left(\mathbf{0}_{E}\right)=\mathbf{0}_{E}\right)$. When $\Phi=\mathrm{C}(\varphi)$ for a 0 -closure operator $\varphi$ on $\mathcal{P}(E)$, then $\Phi^{\prime}$ will be the restriction to $\Pi(E)$ of $\mathrm{C}\left(\varphi_{s}\right)$, where $\varphi_{s}$ is the 0 -closure operator on $\mathcal{P}(E)$ given by $\varphi_{s}(p)=\{p\}$ for all $p \in E$, and $\varphi_{s}(X)=\varphi(X)$ for any non-singleton $X \subseteq E$; here $\operatorname{lnv}\left(\varphi_{s}\right)=\operatorname{lnv}(\varphi) \cup\{\{p\} \mid$ $p \in E\}$ : adding all singletons to a 0 -closure system on $\mathcal{P}(E)$ yields a 0 -closure system. Note that $\mathrm{C}\left(\varphi_{s}\right)\left(\mathbf{0}_{E}\right)=\mathbf{0}_{E}$.

\section{Discussion And CONCLUSION}

We have shown that for a 0 -closure system $\mathcal{S}$ on $\mathcal{P}(E), \nabla^{*}(\mathcal{S})$ and $\nabla(\mathcal{S})$ are closure systems on $\Pi^{*}(E)$ and $\Pi(E)$ respectively, and conversely every $\mathcal{S} \in$ $\mathcal{P}_{0}(\mathcal{P}(E))$ such that $\nabla^{*}(\mathcal{S})$ is a closure system on $\Pi^{*}(E)$, must be a 0 -closure system. The dual problem, namely characterizing when $\nabla^{*}(\mathcal{S})$ and $\nabla(\mathcal{S})$ are kernel systems (i.e., stable under the supremum operation) has also been studied.

Let $\mathcal{S} \in \mathcal{P}_{0}(\mathcal{P}(E))$; we say that $\mathcal{S}$ is a partial connection on $\mathcal{P}(E)$ [20] if for any $\mathcal{B} \subseteq \mathcal{S}, \cap \mathcal{B} \neq \emptyset \Rightarrow \cup \mathcal{B} \in \mathcal{S}$; in other words, any set formed by chaining elements of $\mathcal{S}$ must belong to $\mathcal{S}$. It is easy to see that $\mathcal{S} \in \mathcal{P}_{0}(\mathcal{P}(E))$ is a partial connection iff it satisfies the following two conditions: 
(a) for any $C, D \in \mathcal{S}, C \cap D \neq \emptyset \Rightarrow C \cup D \in \mathcal{S}$;

(b) for any directed subset $\mathcal{D}$ of $\mathcal{S}, \cup \mathcal{D} \in \mathcal{S}$.

When the partial connection $\mathcal{S}$ contains all singletons $\{p\}, p \in E$, it is called a connection on $\mathcal{P}(E)$; the latter concept originated in [27]. Note that any connection $\mathcal{S}$ satisfies the condition $\Delta(\nabla(\mathcal{S}))=\mathcal{S}$; indeed, for any $C \in \mathcal{S} \backslash\{0\}$, $\{C\} \cup\{\{p\} \mid p \in E \backslash C\} \in \nabla(\mathcal{S})$, so $C \in \bigcup \nabla(\mathcal{S}) \subseteq \Delta(\nabla(\mathcal{S})$ ). Now for $\mathcal{S} \in \mathcal{P}_{0}(\mathcal{P}(E))$, we have:

(1) $\nabla^{*}(\mathcal{S})$ is a kernel system on $\Pi^{*}(E)$ iff $\mathcal{S}$ is a partial connection on $\mathcal{P}(E)$ $[20]$.

(2) $\nabla(\mathcal{S})$ is a kernel system on $\Pi(E)$ iff $\mathcal{S}$ is a connection on $\mathcal{P}(E)$ [28].

Combining these two results with Theorems 2 and 6 and the fact that a connection $\mathcal{S}$ satisfies $\Delta(\nabla(\mathcal{S}))=\mathcal{S}$, we deduce:

(3) $\nabla^{*}(\mathcal{S})$ is a complete sublattice of $\Pi^{*}(E)$ iff $\mathcal{S}$ is both a partial connection and a 0 -closure system on $\mathcal{P}(E)$.

(4) $\nabla(\mathcal{S})$ is a complete sublattice of $\Pi(E)$ iff $\mathcal{S}$ is both a connection and a 0 -closure system on $\mathcal{P}(E)$.

Note that in item 4 , the corresponding 0 -closure operator $\varphi$ satisfies $\varphi(p)=\{p\}$ for all $p \in E$; this property was called $\mathrm{T}_{1}$ in [17], it is a particular case of the properties given in Lemma 4 ; thus $\mathcal{S}$ is a P-0-closure system. In order to consider Lemma 4 in the general case of an arbitrary partition of atoms, item 4 can be extended as follows:

(5) Let $\pi_{0} \in \Pi(E)$ and let $\mathcal{S} \in \mathcal{P}_{0}(\mathcal{P}(E))$ such that each element of $\mathcal{S}$ is a union of some blocks of $\pi_{0}$; then $\nabla(\mathcal{S})$ is a complete sublattice of $\left\{\pi \in \Pi(E) \mid \pi_{0} \leq \pi\right\}$ iff $\mathcal{S}$ is both a partial connection and a 0-closure system on $\mathcal{P}(E)$, where the blocks of $\pi_{0}$ are the atoms of $\mathcal{S}$.

Items 4 and 5 are slight extensions of Corollary 2.h and Theorem 2.f of [17], where $\mathcal{S}$ was assumed a priori to be a 0 -closure system; the fact that $\mathcal{S}$ is a partial connection was expressed through conditions (a) and (b) above; note that a closure system satisfying property (b) is called algebraic, while property (a) was called Q. Finally, Corollary 2.g of [17] showed that for a P-0-closure system $\mathcal{S}, \nabla(\mathcal{S})$ is stable under binary join iff $\mathcal{S}$ satisfies property (a); the proof is similar to that of item 2 in [28].

Let us conclude. We have analysed the relation between closure systems on sets and closure systems on (partial) partitions, where the closed sets are the blocks of the closed (partial) partitions; it is induced by the two adjunctions $(\nabla, \Delta)$ and $\left(\nabla^{*}, \Delta^{*}\right)$. We generalize the analysis made by Jordens and Sturm $[16,17]$ in two ways: first we give stronger results in the case of partitions, second we extend the framework to partial partitions. We have also considered the design of the associated closure operator on partial partitions by iterating the application to the blocks of the corresponding closure operator on sets. 
The topic of closure operators on partitions and partial partitions is relevant in applied domains such as data clustering and image segmentation. Besides those based on applying a closure operator to the blocks, there are many other closure operators on partial partitions, in particular those that cluster together blocks on the basis of proximity relations [21].

\section{REFERENCES}

[1] BARTHÉLEMY, J. P.-LECLERC, B.: The median procedure for partitions. In: Partitioning Data Sets (I.J. Cox, P. Hansen, and B. Julesz, eds.), DIMACS Series in Discrete Mathematics and Theoretical Computer Science, Vol. 19, American Mathematical Society, Providence, R.I., 1995, pp. 3-34.

[2] BAUER, A.-BIRKEDAL, L.-SCOTT, D. S.: Equilogical spaces, Theoretical Computer Science 315 (2004), 35-59.

[3] BIRKHOFF, G.: Lattice Theory, American Mathematical Society Colloquium Publications, Vol. 25, American Mathematical Society, Providence, RI, 8th printing, 3rd edition, 1995.

[4] BLOCH, I.-HEIJMANS, H.-RONSE, C.: Mathematical Morphology. In: Handbook of Spatial Logics (M. Aiello, I. Pratt-Hartmann, and J. van Benthem, eds.), Springer, 2007, Chapter 14, pp. 857-944.

[5] BLYTH, T. S.: Lattices and Ordered Algebraic Structures, Springer, London, 2005.

[6] DAVEY, B. A.-PRIESTLEY, H. A.: Introduction to Lattices and Order, Cambridge University Press, Cambridge, UK, 2nd edition, 2002.

[7] DENECKE, K.-ERNÉ, M.,-WISMATH, S.L. (EDS.): Galois Connections and Applications, Kluwer, Dordrecht, NL, 2004

[8] DRAS̆KOVIČOVÁ, H.: The lattice of partitions in a set, Acta Fac. Rerum Natur. Univ. Comenian. Math. Publ. 24 (1970), 37-65.

[9] DRAS̆KOVIC̆OVÁ, H.: Congruence relations on the lattice of partitions in a set, Matematický C̆asopis (Slovenska Akademia Vied) 21 (1971), 141-153.

[10] ERNÉ, M.: Closure. In: Beyond Topology (F. Mynard and E. Pearl, eds.), Contemporary Mathematics, Vol. 486, American Mathematical Society, 2009, pp. 163-238.

[11] GANTER, B.-WILlE, R.: Formal Concept Analysis. In: General Lattice Theory (G. Grätzer, ed.), Birkhäuser, Basel, 2nd edition, 2003, Appendix H, pp. 591-605.

[12] GIERZ, G.-HOFMANN, K. H.-KEIMEL, K.-LAWSON, J. D.-MISLOVE, M.SCOTT, D. S.: Continuous Lattices and Domains, Encyclopedia of Mathematics and its Applications, Vol. 93, Cambridge University Press, Cambridge, UK, 2003.

[13] GORDON, A. D.-VICHI, M.: Partitions of partitions, Journal of Classification 15 (1998), 265-285.

[14] HEIJMANS, H. J. A. M.: Morphological Image Operators, Advances in Electronics and Electron Physics Series, Academic Press, Boston, 1994.

[15] HEIJMANS, H. J. A. M.-SERRA, J.: Convergence, continuity and iteration in mathematical morphology, Journal of Visual Communication and Image Representation 3 (1992), 84-102.

[16] JORDENS, O.-STURM, T.: Closure systems of equivalences with a local property, Mathematica Japonica 36 (1991), 245-250.

[17] JORDENS, O.-STURM, T.: Equivalences with closed equivalence classes, Mathematica Japonica 36 (1991), 291-304.

[18] MITCHELL, J. C.: Foundations for Programming Languages, MIT Press, 1996.

[19] ORE, O.: Theory of equivalence relations, Duke Mathematical Journal 9 (1942), 573-627. 
[20] RONSE, C.: Partial partitions, partial connections and connective segmentation, Journal of Mathematical Imaging and Vision 32 (2008), 97-125.

[21] RONSE, C.: Adjunctions on the lattices of partitions and of partial partitions, Applicable Algebra in Engineering, Communication and Computing, 21 (2010), 343-396.

[22] RONSE, C.: Idempotent block splitting on partial partitions, I: isotone operators, Order 28 (2011), 273-306.

[23] RONSE, C.: Idempotent block splitting on partial partitions, II: non-isotone operators, Order 28 (2011), 307-339.

[24] RONSE, C.-SERRA, J.: Algebraic foundations of morphology. In: Mathematical morphology:From Theory to Applications (L. Najman and H. Talbot, eds.), ISTE / J. Wiley \& Sons, 2010, Chapter 2, pp. 35-80.

[25] SABELFELD, A.-SANDS, D.: A per model of secure information flow in sequential programs, Higher-Order and Symbolic Computation 14 (2001), 59-91.

[26] SERRA, J. (ed.): Image Analysis and Mathematical Morphology, II: Theoretical Advances, Academic Press, London, 1988.

[27] SERRA, J.: Mathematical morphology for Boolean lattices. In: Image Analysis and Mathematical Morphology, II: Theoretical Advances (J. Serra, ed.), Academic Press, London, 1988, Chapter 2, pp. 37-58.

[28] SERRA, J.: A lattice approach to image segmentation, Journal of Mathematical Imaging and Vision 24 (2006), 83-130.

[29] STURM, T.: Verbände von Kernen isotoner Abbildungen, Czekoslovak Mathematical Journal 22(97) (1972), 126-144.

LSIIT UMR 7005 CNRS-UdS, Parc D'Innovation, Boulevard SÉbastien Brant, BP 10413, 67412 ILLKIRCH CEDEX, FRANCE

E-mail address: cronse@unistra.fr - http://lsiit-miv.u-strasbg.fr/ 